\title{
Nonlinear Vibrational Analysis of Nanobeams Embedded in an Elastic Medium including Surface Stress Effects
}

\author{
S. Azizi, ${ }^{1}$ B. Safaei, ${ }^{2}$ A. M. Fattahi, ${ }^{3}$ and M. Tekere ${ }^{1}$ \\ ${ }^{1}$ Department of Environmental Sciences, School of Agriculture and Environmental Sciences, University of South Africa, \\ P.O. Box 1710, Florida, South Africa \\ ${ }^{2}$ Young Researchers and Elite Club, Electronic Branch, Islamic Azad University, Tehran, Iran \\ ${ }^{3}$ Department of Mechanical Engineering, Tabriz Branch, Islamic Azad University, Tabriz, Iran \\ Correspondence should be addressed to A. M. Fattahi; a.fattahi@iaut.ac.ir
}

Received 26 July 2015; Revised 16 November 2015; Accepted 22 November 2015

Academic Editor: Santiago Garcia-Granda

Copyright $\odot 2015$ S. Azizi et al. This is an open access article distributed under the Creative Commons Attribution License, which permits unrestricted use, distribution, and reproduction in any medium, provided the original work is properly cited.

Due to size-dependent behavior of nanostructures, the classical continuum models are not applicable for the analyses at this submicron size. Surface stress effect is one of the most important matters which make the nanoscale structures have different properties compared to the conventional structures due to high surface to volume ratio. In the present study, nonlinear free vibrational characteristics of embedded nanobeams are investigated including surface stress effects. To this end, a thin surface layer is assumed on the upper and lower surfaces of the cross section to separate the surface and bulk of nanobeams with their own different material properties. Based on harmonic balance method, closed-form analytical solution is conducted for nonlinear vibrations to obtain natural frequencies of embedded nanobeams with and without considerations of surface elasticity and residual surface tension effects corresponding to the various values of nondimensional amplitude, elastic foundation modulus, and geometrical variables of the system. Selected numerical results are given to indicate the influence of each one in detail.

\section{Introduction}

Because of advancing science and technology, there has been quite request for new materials with extraordinary properties for implementation in various emerging fields of development. Due to a wide range of applications nanostructures and materials at nanoscale have been attracting consequential interest in different aspects of nanoscience and nanotechnology.

For example, $\mathrm{Xu}$ and his assistants [1] explored the $\mathrm{PH}-$ sensitive property of the single-walled carbon nanotubes (SWCNTs) based on electroactive group on SWCNT by different pulse voltammetry technique to show the broad biosensor application of carbon nanotube in electrochemical area. Wu et al. [2] investigated the resonant frequency shift of the fixed-free SWCNTs caused by the addition of a nanoparticle to the beam tip using a beam-bending model to explore the suitability of the SWCNT as a mass detector device. A nanocomposite film of embedded SWCNTs was prepared by Penza et al. [3] to develop highly sensitive microacoustic vapor sensors based on surface acoustic waves. They indicated that SWCNT nanocomposite vapor sensitivity results in being significantly enhanced with respect to traditional organic molecular cavities materials.

The surface stress effect can be easily observed at the atomic scale due to high surface to volume ratio, and this has been clearly indicated and explained $[4,5]$. The main reason for the phenomenon is based on different environment conditions for the atoms which are near free surface compared to the ones at the bulk of the material. For conventional structures in which the number of atoms near free surface is so negligible relative to the total number of atoms of the system, stress effects on surface physics are unimportant and can be neglected. However, in the case of nanostructures, through increasing ratio of surface area and volume these effects can make considerable influence. 
There are so many computational and experimental researches in which the influence of surface stress effects on behavior of the structures at nanoscale has been studied. Gurtin et al. [6] analyzed the effect of residual surface tension on the stress concentration around a nanosized spheroidal cavity in an isotropic medium based on surface elasticity. Radi [7] described the material behavior with the indeterminate theory of couple surface elasticity to investigate the problem of a stationary semi-infinite crack in an elastic solid. The effect of surface/interface stress on the macroscopic plastic behaviors of nanocomposites has been investigated by Zhang et al. [8] through a new second-order moment nonlinear micromechanics theory.

Gurtin et al. [6] presented a model for vibrational analysis of microbeams including surface elasticity influences represented by applying distributed traction and compressive axial force. Then, for an isotropic surface, Gurtin and Ian Murdoch [9] proposed a continuum model of surface elasticity in which the Laplace-Young equation extended to solid materials. The model has the capability to incorporate the surface stress effects into the mechanical response of nanostructures which has been applied in many studies conducted for various problems about mechanical behavior of the structures at nanoscale [10-18].

A new frontier of research in the area of computational nanomechanics is to study the behavior of structures at very small length scales. Then Ansari and Sahmani [19] proposed a nonclassical solution to analyze bending and buckling responses of nanobeams including surface stress effects. Explicit formulas are proposed relevant to each type of beam theory to evaluate the surface stress effects on the displacement profile and critical buckling load of the nanobeams. Ansari and Sahmani [20] presented the free vibration characteristics of nanoplates including surface stress effects are investigated based on the continuum modeling approach. To this end, Gurtin-Ian Murdoch continuum elasticity approach is incorporated into the different types of plate theory, namely, classical plate theory (CLPT) and firstorder shear deformation theory (FSDT) to develop nonclassical continuum plate models for free vibration analysis of the nanoplates including surface stress effects.

Karličić et al. [21] proposed the stochastic parametric vibrations of a Voigt-Kelvin nanobeam based on Eringen's nonlocal elasticity theory of the Helmholtz and bi-Helmholtz type of kernel. The axial force consists of a constant part and a time-dependent stochastic function. By using the direct Lyapunov method, bounds of almost sure asymptotic stability of a viscoelastic nanobeam are obtained as a function of retardation time, variance of the stochastic force, geometric ratio, scale coefficients, and intensity of the deterministic component of axial loading.

In this work, the surface stress effects including both surface elasticity and residual surface tension are incorporated into the nonlinear free vibrations of nanobeams embedded in an elastic medium based on the model of Gurtin et al. [6]. Selected numerical results are presented to indicate the influence of nondimensional amplitude, elastic foundation modulus, and geometrical variables of the system on the value of surface stress effects.

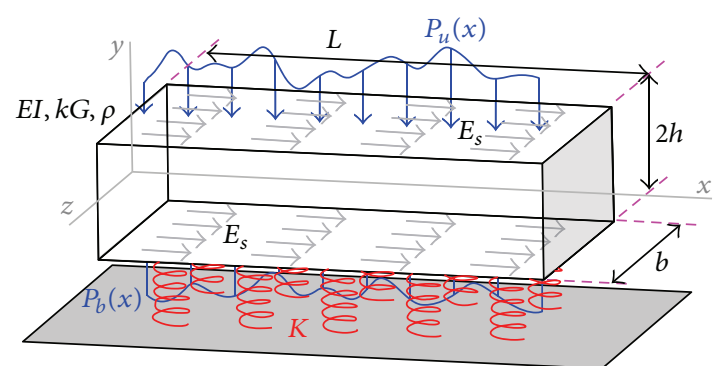

FIGURE 1: Geometry and properties of the considered problem.

\section{Surface Elasticity Model for Nanobeams}

Consider a straight uniform beam with the length $L$ and rectangular cross section of thickness $2 h$. A coordinate system $(x, y$, and $z)$ is introduced on the central axis of the beam, whereas $x$-axis is taken along the length of the beam, $y$-axis is taken in the width direction, and $z$-axis is taken along the depth (height) direction. Also, the origin of the coordinate system is selected at the left end of the beam (Figure 1). It is assumed that the deformations of the beam take place in the $x-z$ plane, so the displacement components $\left(u_{1}, u_{2}\right.$, and $\left.u_{3}\right)$ along the axes $(x, y$, and $z)$ are only dependent on the $x$ and $z$ coordinates and time $t$.

To represent the surface stress effects in this work, thin surface layers with surface elastic modulus $E_{s}$ are assumed on the upper and lower surfaces of the cross section to separate the surfaces and the bulk of nanobeams. According to the Laplace-Young equation, the upper $\left(\tau_{u}\right)$ and lower $\left(\tau_{b}\right)$ residual surface tension can be considered as distributed loading on surfaces [6] which can be expressed in the following forms:

$$
\begin{aligned}
& p_{u}(x)=\tau_{u} b \frac{\partial^{2} w}{\partial x^{2}}, \\
& p_{b}(x)=\tau_{b} b \frac{\partial^{2} w}{\partial x^{2}},
\end{aligned}
$$

where $w$ is the transverse displacement of the beam, $b$ denotes the width of nanobeam, and $\tau_{u}$ and $\tau_{b}$ stand for the residual surface stresses on the upper and lower surfaces of nanobeam, respectively.

Furthermore, based on the Winkler elastic foundation model [22] the normal pressure of elastic medium is simulated as a series of closely spaced, vertical linear elastic springs. Thereupon, the loading corresponding to this type of foundation can be expressed as

$$
p_{e}(x)=-K w
$$

where $K$ is the Winkler modulus parameter corresponding to the normal pressure.

On the basis of energy method, the total potential energy of the system contains the elastic strain energy of the bulk and surfaces. Through implementing generalized Hamilton's principle, the governing differential equation for nonlinear 
free vibrations of Euler-Bernoulli nanobeam embedded in an elastic medium is obtained as

$$
\begin{aligned}
(E I+ & \left.+2 E_{s} b h^{2}\right) \frac{\partial^{4} w(x, t)}{\partial x^{4}}-\left(\tau_{u}+\tau_{b}\right) b \frac{\partial^{2} w(x, t)}{\partial x^{2}} \\
& +K w(x, t)+\rho A \frac{\partial^{2} w(x, t)}{\partial t^{2}} \\
= & \left(\frac{E A}{2 L} \int_{0}^{L}\left(\frac{\partial w(x, t)}{\partial x}\right)^{2} d x\right) \frac{\partial^{2} w(x, t)}{\partial x^{2}},
\end{aligned}
$$

where $E$ and $E_{s}$ are the bulk and surface Young's modulus, respectively.

The transverse displacement $w$ can be considered in the following generalized form which satisfies the simply supported boundary conditions:

$$
w(x, t)=W_{m}(t) \sin \frac{m \pi x}{L} .
$$

Substituting (4) into the governing equation of (3) yields

$$
\begin{aligned}
& \frac{d^{2} W_{m}(t)}{d t^{2}}+\left[\left(\frac{m \pi}{L}\right)^{4} \frac{E I}{\rho A}+\left(\frac{m \pi}{L}\right)^{4} \frac{2 E_{s} b h^{2}}{\rho A}\right. \\
& \left.+\left(\frac{m \pi}{L}\right)^{2} \frac{\left(\tau_{u}+\tau_{b}\right) b}{\rho A}+\frac{K}{\rho A}\right] W_{m}(t) \\
& +\frac{1}{4} \frac{E}{\rho}\left(\frac{m \pi}{L}\right)^{4} W_{m}^{3}(t)=0
\end{aligned}
$$

in which $m$ is the vibrational mode number, $\rho$ denotes the mass density, and $A$ and $I$ are cross-sectional area and moment of inertia of the nanobeam, respectively.

The transformations are considered as follows:

$$
\begin{aligned}
r & =\sqrt{\frac{L}{A}}, \\
X_{m} & =\frac{W_{m}}{r}, \\
\tau & =\tau_{u}+\tau_{b}, \\
\mu & =\omega t, \\
\omega_{L} & =\left(\frac{m \pi}{L}\right)^{2} \sqrt{\frac{E I}{\rho A}}, \\
\omega_{K} & =\sqrt{\frac{K}{\rho A}}, \\
\omega_{E} & =\left(\frac{m \pi}{L}\right)^{2} \sqrt{\frac{2 E_{s} b h^{2}}{\rho A}}, \\
\omega_{\tau} & =\frac{m \pi}{L} \sqrt{\frac{\left(\tau_{u}+\tau_{b}\right) b}{\rho A} .}
\end{aligned}
$$

Now (5) can be expressed in the following dimensionless nonlinear equation:

$$
\begin{gathered}
\left(\frac{\omega}{\omega_{l}}\right)^{2} \ddot{X}_{m}+\left(1+\left(\frac{\omega_{E}}{\omega_{l}}\right)^{2}+\left(\frac{\omega_{\tau}}{\omega_{l}}\right)^{2}+\left(\frac{\omega_{k}}{\omega_{l}}\right)^{2}\right) X_{m} \\
+\alpha X_{m}^{3}=0,
\end{gathered}
$$

in which $\alpha=1 / 4$.

The harmonic balance method is utilized to solve the nonlinear equations given by (7). Based on this method, it is assumed that the solution can be represented as a truncated Fourier series of the form

$$
X_{m}(\mu)=A_{m} \cos \mu+A_{m+n} \cos 3 \mu .
$$

Applying the assumed solution to (7) in conjunction with expanding each term as a Fourier series leads to throwing out higher harmonics other than the ones included in the original assumed solution. After equating the coefficients like cosine harmonics (such as the $\cos \tau$ and $\cos 3 \tau$ terms), one can arrive at a set of algebraic equations of this form

$$
\begin{aligned}
& -\left(\frac{\omega}{\omega_{l}}\right)^{2} A_{m}+\left(1+\left(\frac{\omega_{E}}{\omega_{l}}\right)^{2}+\left(\frac{\omega_{\tau}}{\omega_{l}}\right)^{2}+\left(\frac{\omega_{k}}{\omega_{l}}\right)^{2}\right) A_{m} \\
& +\frac{3}{4} \alpha A_{m}^{2} A_{m+n}+\frac{3}{2} \alpha A_{m+n}^{2} A_{m}+\frac{3}{4} \alpha A_{m}^{3}=0 \\
& -9\left(\frac{\omega}{\omega_{l}}\right)^{2} A_{m+n} \\
& +\left(1+\left(\frac{\omega_{E}}{\omega_{l}}\right)^{2}+\left(\frac{\omega_{\tau}}{\omega_{l}}\right)^{2}+\left(\frac{\omega_{k}}{\omega_{l}}\right)^{2}\right) A_{m+n} \\
& \quad+\frac{3}{2} \alpha A_{m}^{2} A_{m+n}+\frac{3}{4} \alpha A_{m+n}^{3}+\frac{1}{4} \alpha A_{m}^{3}=0 .
\end{aligned}
$$

The preceding nonlinear equations can be solved for $\omega, A_{m+n}$ using the Newton-Raphson numerical scheme by choosing $A_{m}$ as a determined parameter.

\section{Numerical Results and Discussion}

In this section, selected numerical results are presented to show the influence of different parameters on the nonlinear vibrational response of nanobeams embedded in an elastic medium including surface stress effects. Anodic alumina with two surface crystal orientations of [ $\left[\begin{array}{lll}1 & 0 & 0\end{array}\right]$ and $\left[\begin{array}{lll}1 & 1 & 1\end{array}\right]$ is considered in the analytical solution. The material properties and related surface parameters for each crystal orientation are given in Table 1.

3.1. Effect of Elastic Foundation Modulus on Nonlinear Frequency Ratio. To explore the effect of surrounding elastic medium on the nonlinear vibrations of nanobeams, variation of $\omega_{\text {nonlinear with surface stress effects }} / \omega_{\text {linear with surface stress effects }}$ with elastic foundation modulus for $\left[\begin{array}{lll}1 & 0 & 0\end{array}\right]$ and $\left[\begin{array}{lll}1 & 1 & 1\end{array}\right]$ crystal orientations is illustrated in Figures 2(a) and 2(b), respectively. In both cases it is assumed that $L=30 \mathrm{~nm}$ and 
TABLE 1: Mechanical properties of anodic alumina.

\begin{tabular}{lccccc}
\hline Crystal orientation & $\begin{array}{c}\text { Young's modulus } \\
(\mathrm{GPa})\end{array}$ & Poisson's ratio & $\begin{array}{c}\text { Mass density } \\
\left(\mathrm{Kg} / \mathrm{m}^{3}\right)\end{array}$ & $\begin{array}{c}\text { Surface elastic } \\
\text { modulus (N/m) }\end{array}$ & $\begin{array}{c}\text { Residual } \\
\text { surface tension } \\
(\mathrm{N} / \mathrm{m})\end{array}$ \\
\hline$\left[\begin{array}{lll}1 & 0 & 0\end{array}\right]$ & 70 & 0.3 & 2700 & -7.9253 & 0.5689 \\
{$\left[\begin{array}{lll}1 & 1\end{array}\right]$} & 70 & 0.3 & 2700 & 5.1882 & 0.9108 \\
\hline
\end{tabular}

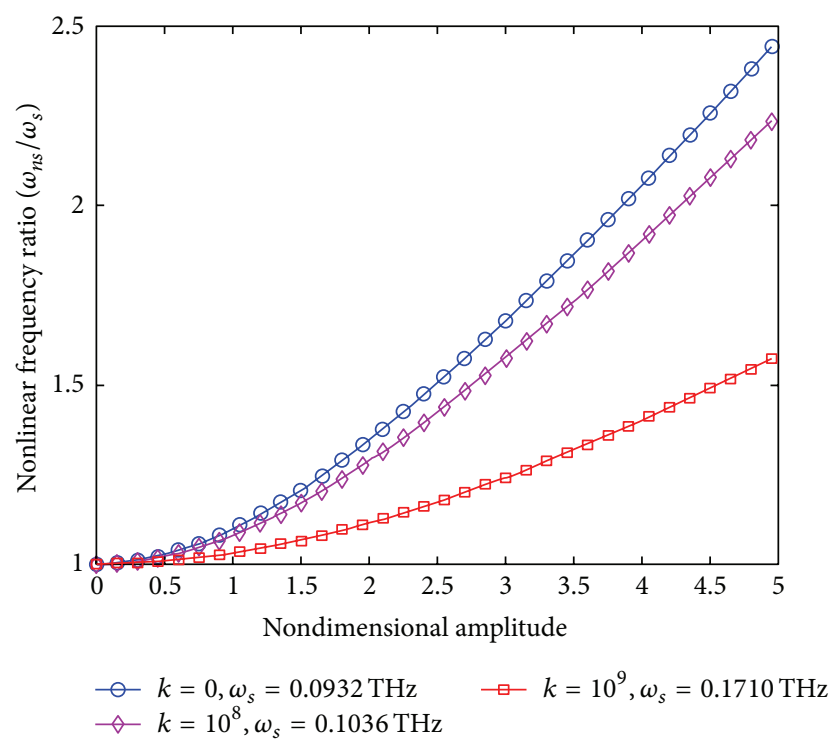

(a)

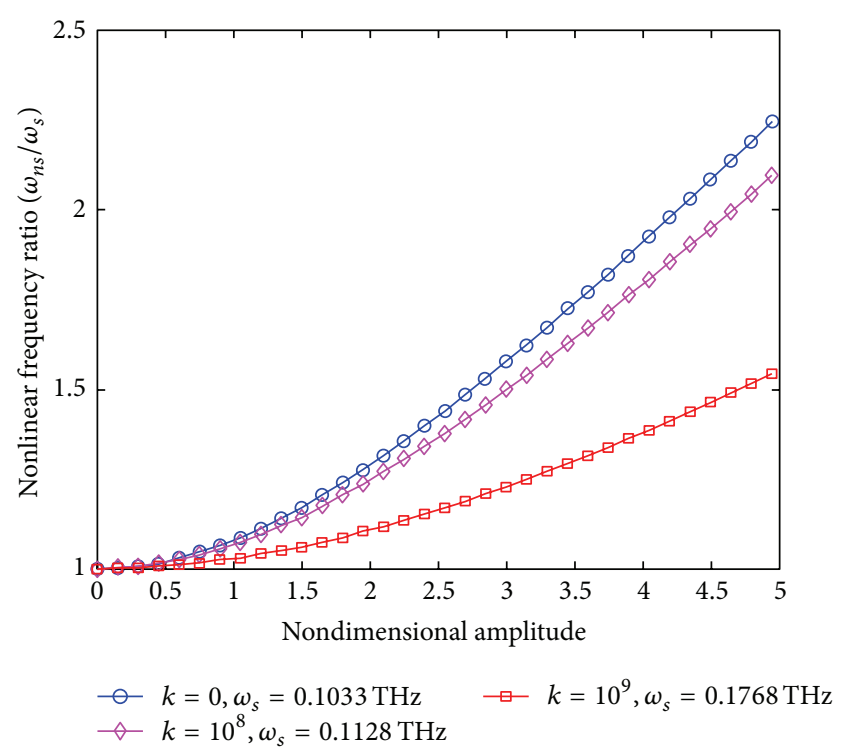

(b)

FIGURE 2: Effect of elastic foundation modulus on nonlinear frequency response for (a) $\left[\begin{array}{lll}1 & 0 & 0\end{array}\right]$ crystal orientation, (b) [ $\left[\begin{array}{ll}1 & 1\end{array}\right]$ crystal orientation.

$h=b=0.1 L$. It can be observed that, for both crystal orientations, the difference between linear and nonlinear responses of nanobeams increases with increase of nondimensional amplitude of vibration which is more prominent for lower values of elastic foundation modulus. In other words, the elastic medium makes the effect of nonlinearity on the vibrational behavior of nanobeams less important.

3.2. Effect of Length of Nanobeam on Nonlinear Frequency Ratio. The effect of length of nanobeams on the nonlinear free vibrational analysis is shown in Figure 3. In this figure, the value of $\omega_{\text {nonlinear with surface stress effects }} /$ $\omega_{\text {linear with surface stress effects }}$ is plotted against the length of the embedded nanobeam corresponding to different elastic foundation modulus and crystal orientations. It can be seen that, for both crystal orientations and all values of elastic foundation moduli, the increase of length of nanobeams leads to decrease of the effect of nonlinearity inclusion. Also, it is shown that, for the higher value of elastic foundation

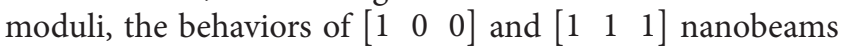
are approximately the same. However, for the lower value of $K$, the difference between the responses of two nanobeams with different crystal orientations is considerable especially for lower values of length.
Additionally, for the case of [ $\left[\begin{array}{lll}1 & 1 & 1\end{array}\right]$ crystal orientation, it is seen that, for the higher elastic medium $\left(K=10^{8}\right)$, by increasing the length of nanobeam, the effect of surface stress decreases with lower slope, especially for shorter nanobeams.

3.3. Effect of Height of Nanobeam on the Frequency Ratio. To see the effect of height of nanobeams on the nonlinear vibrational response, the variation of $\omega_{\text {nonlinear with surface stress effects } /}$ $\omega_{\text {nonlinear without surface stress effects }}$ with respect to the height of embedded nanobeams relevant to various elastic foundation moduli is depicted in Figure 4 for both $\left[\begin{array}{lll}1 & 0 & 0\end{array}\right]$ and $\left[\begin{array}{lll}1 & 1 & 1\end{array}\right]$ crystal orientations.

It is observed that, in the case having no elastic medium $(K=0)$, the value of frequency ratio increases for all values of height. However, with the inclusion of elastic medium, the behavior of nanobeam changes totally as the height of nanobeam can make increase or decrease of the frequency ratio at different values. Furthermore, in the case of $\left[\begin{array}{lll}1 & 0 & 0\end{array}\right]$ crystal orientation, a critical value for the height of nanobeam is found corresponding to which the surface stress effect will be the same for all values of elastic foundation modulus.

Also, for both crystal orientations, it is observed that, for $K=10^{7}$, by increasing the height of nanobeam, the effect of surface stress increases first and then decreases. However, 


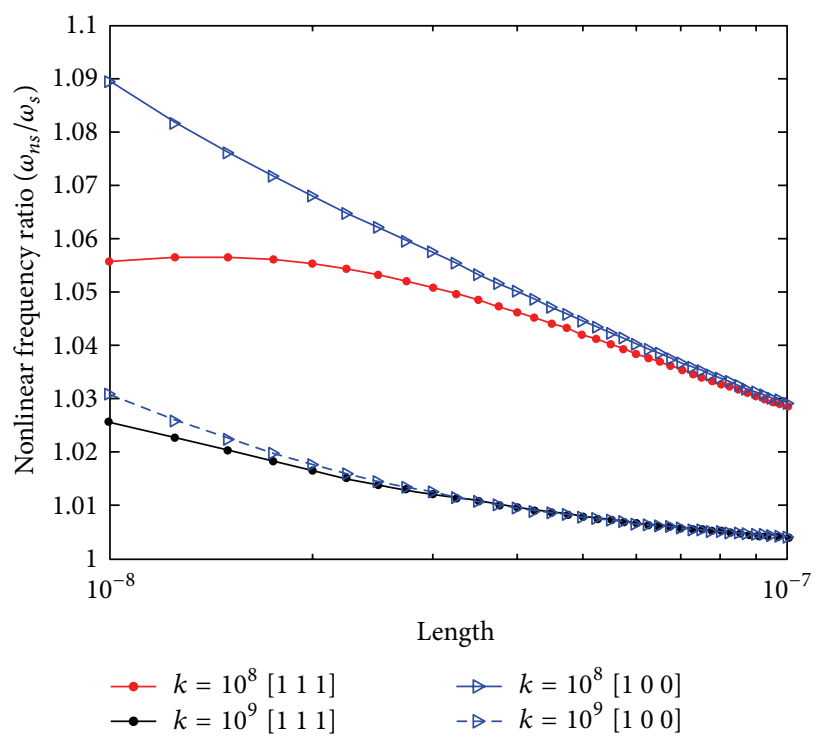

FIGURE 3: Effect of length of the nanobeams on nonlinear frequency response.

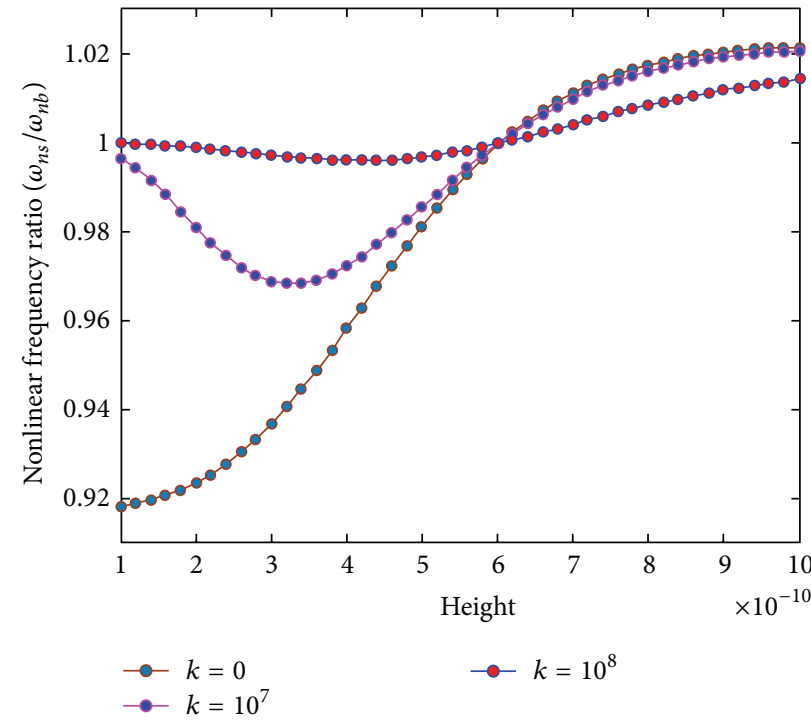

(a)

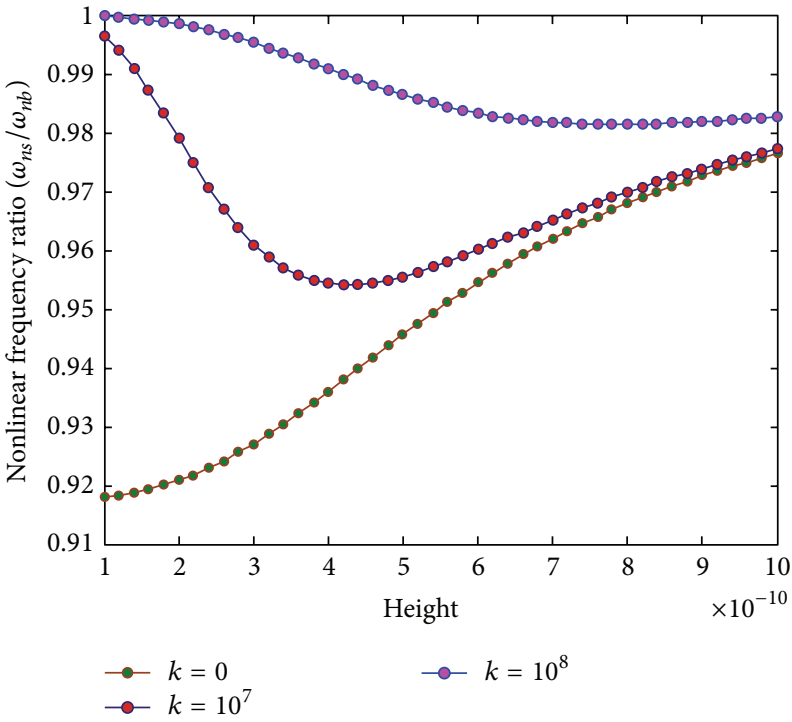

(b)

FIGURE 4: Effect of height of nanobeams on nonlinear frequency response corresponding to different values of elastic foundation modulus:

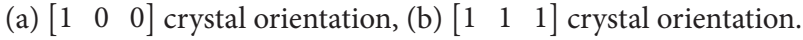

for a stiffer elastic medium $\left(K=10^{8}\right)$, the surface stress effect decreases continuously by increasing the height of nanobeam. In other words, for a medium value of elastic medium, in order to decrease the effect of surface stress, it is necessary to increase the height of nanobeam above a special value. However, for high elastic medium, any increase in the value of beam height leads to lower surface stress effect.

Figure 5 indicates the influence of height of nanobeams on the value of $\omega_{\text {nonlinear with surface stress effects } /}$ $\omega_{\text {nonlinear without surface stress effects }}$ relevant to different amounts of nondimensional amplitude of vibrations for $\left[\begin{array}{lll}1 & 0 & 0\end{array}\right]$ and $\left[\begin{array}{lll}1 & 1 & 1\end{array}\right]$ crystal orientations. It can be found that, in both types of crystal orientation, the height of nanobeam decreases the frequency ratio until a certain value of height. Beyond this certain height the value of nonlinear frequency ratio increases. It is seen that this pattern of variation of the frequency ratio is more sensible corresponding to higher values of nondimensional amplitude.

Also, it can be observed that, for the case of [ $\left[\begin{array}{lll}1 & 0 & 0\end{array}\right]$ crystal orientation, the values of frequency ratio corresponding to a certain value of height are equal for all nondimensional amplitudes. In other words, there is an amount of the height for the nanobeam with $\left[\begin{array}{lll}1 & 0 & 0\end{array}\right]$ crystal orientation relevant to which the surface stress effect is the same for all values of nondimensional amplitude. 


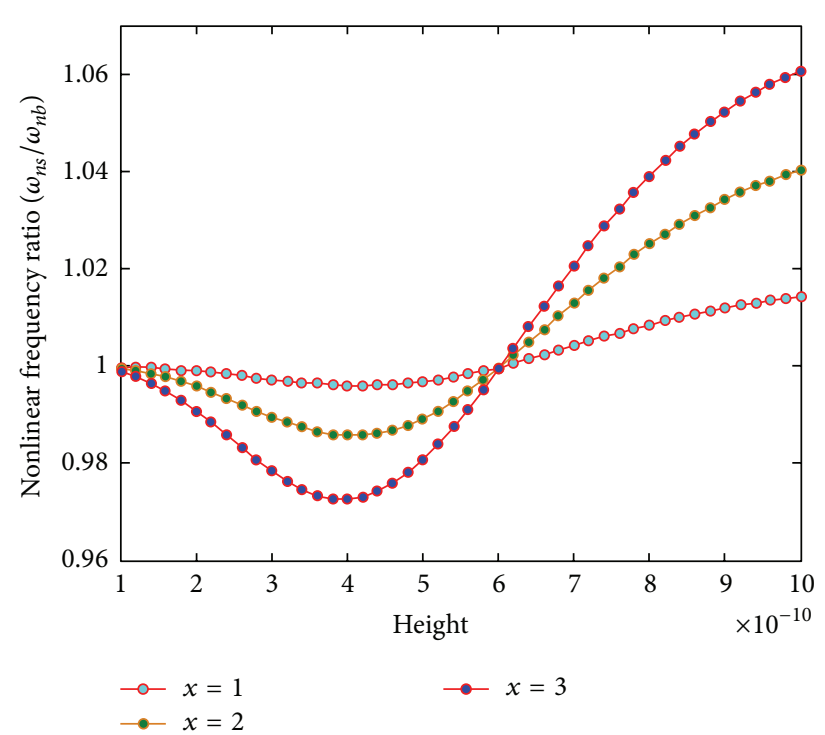

(a)

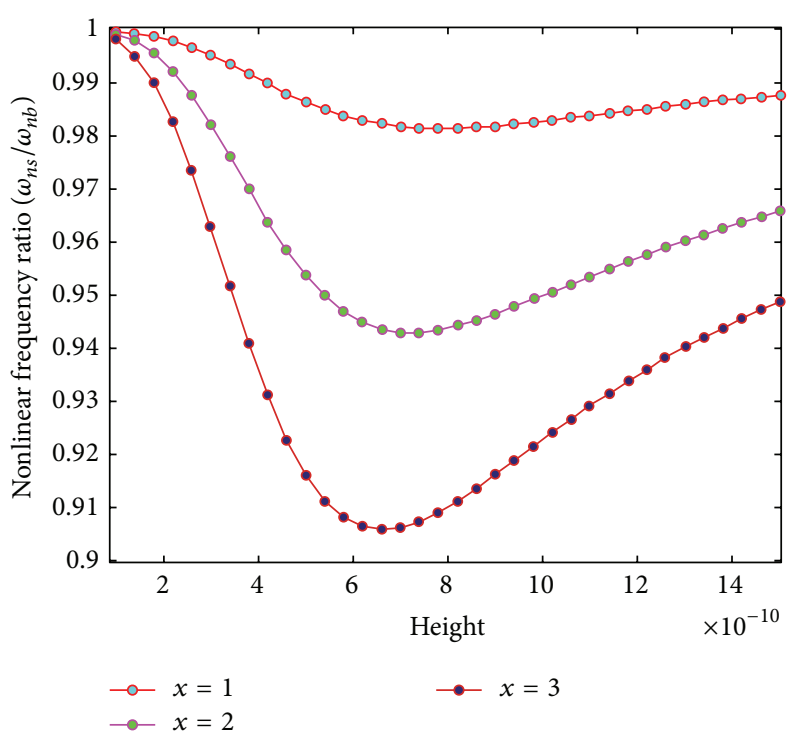

(b)

FIGURE 5: Effect of height of the nanobeams on nonlinear frequency response corresponding to different values of nondimensional amplitude: (a) $\left[\begin{array}{lll}1 & 0 & 0\end{array}\right]$ crystal orientation, (b) [ $\left.\begin{array}{lll}1 & 1 & 1\end{array}\right]$ crystal orientation.

\section{Conclusion}

In this work, nonlinear vibrational characteristics of embedded nanobeams in an elastic medium are investigated including surface stress effects. To this end, both surface elasticity and residual surface tension effects are incorporated into the nonlinear vibrational analysis of embedded Euler-Bernoulli nanobeam.

Analytical solution is presented for simply supported nanobeams and selected numerical results are given to show the influences of nondimensional amplitude, elastic foundation modulus, and geometrical variables of the system in detail corresponding to two types of crystal orientations. It is found that surface stress effect can have significant influence on the nonlinear vibrational behavior of nanobeams and this effect varies relevant to different parameters of the system.

\section{Conflict of Interests}

The authors declare that there is no conflict of interests regarding the publication of this paper.

\section{References}

[1] Z. Xu, X. Chen, X. Qu, J. Jia, and S. Dong, "Single-wall carbon nanotube-based voltammetric sensor and biosensor," Biosensors and Bioelectronics, vol. 20, no. 3, pp. 579-584, 2004.

[2] D. H. Wu, W. T. Chien, C. S. Chen, and H. H. Chen, "Resonant frequency analysis of fixed-free single-walled carbon nanotubebased mass sensor," Sensors and Actuators A: Physical, vol. 126, no. 1, pp. 117-121, 2006.

[3] M. Penza, M. A. Tagliente, P. Aversa, G. Cassano, and L. Capodieci, "Single-walled carbon nanotubes nanocomposite microacoustic organic vapor sensors," Materials Science and Engineering C, vol. 26, no. 5-7, pp. 1165-1170, 2006.
[4] F. H. Streitz, R. C. Cammarata, and K. Sieradzki, "Surface-stress effects on elastic properties. I. Thin metal films," Physical Review $B$, vol. 49, no. 15, pp. 10699-10706, 1994.

[5] R. Dingreville, J. Qu, and M. Cherkaoui, "Surface free energy and its effect on the elastic behavior of nano-sized particles, wires and films," Journal of the Mechanics and Physics of Solids, vol. 53, no. 8, pp. 1827-1854, 2005.

[6] M. E. Gurtin, X. Markenscoff, and R. N. Thurston, "Effect of surface stress on the natural frequency of thin crystals," Applied Physics Letters, vol. 29, no. 9, pp. 529-530, 1976.

[7] E. Radi, "On the effects of characteristic lengths in bending and torsion on Mode III crack in couple stress elasticity," International Journal of Solids and Structures, vol. 45, no. 10, pp. 3033-3058, 2008.

[8] W. X. Zhang, T. J. Wang, and X. Chen, "Effect of surface/interface stress on the plastic deformation of nanoporous materials and nanocomposites," International Journal of Plasticity, vol. 26, no. 7, pp. 957-975, 2010.

[9] M. E. Gurtin and A. Ian Murdoch, "Surface stress in solids," International Journal of Solids and Structures, vol. 14, no. 6, pp. 431-440, 1978.

[10] S. Azizi, A. M. Fattahi, and J. T. Kahnamouei, "Evaluating mechanical properties of nanoplatelet reinforced composites under mechanical and thermal loads," Journal of Computational and Theoretical Nanoscience, In press.

[11] G. F. Wang and X. Q. Feng, "Effects of surface stresses on contact problems at nanoscale," Journal of Applied Physics, vol. 101, no. 1, Article ID 013510, 2007.

[12] J. He and C. M. Lilley, "Surface stress effect on bending resonance of nanowires with different boundary conditions," Applied Physics Letters, vol. 93, no. 26, Article ID 263108, 2008.

[13] X. J. Zhao and R. K. Rajapakse, "Analytical solutions for a surface-loaded isotropic elastic layer with surface energy effects," International Journal of Engineering Science, vol. 47, no. 11-12, pp. 1433-1444, 2009. 
[14] E. Gordeliy, S. G. Mogilevskaya, and S. L. Crouch, "Transient thermal stresses in a medium with a circular cavity with surface effects," International Journal of Solids and Structures, vol. 46, no. 9, pp. 1834-1848, 2009.

[15] M. Jammes, S. G. Mogilevskaya, and S. L. Crouch, "Multiple circular nano-inhomogeneities and/or nano-pores in one of two joined isotropic elastic half-planes," Engineering Analysis with Boundary Elements, vol. 33, no. 2, pp. 233-248, 2009.

[16] B. B. On, E. Altus, and E. B. Tadmor, "Surface effects in non-uniform nanobeams: continuum vs. atomistic modeling," International Journal of Solids and Structures, vol. 47, no. 9, pp. 1243-1252, 2010.

[17] H. Sheng, H. Li, P. Lu, and H. Xu, "Free vibration analysis for micro-structures used in MEMS considering surface effects," Journal of Sound and Vibration, vol. 329, no. 2, pp. 236-246, 2010.

[18] S. G. Mogilevskaya, S. L. Crouch, A. L. Grotta, and H. K. Stolarski, "The effects of surface elasticity and surface tension on the transverse overall elastic behavior of unidirectional nanocomposites," Composites Science and Technology, vol. 70, no. 3, pp. 427-434, 2010.

[19] R. Ansari and S. Sahmani, "Bending behavior and buckling of nanobeams including surface stress effects corresponding to different beam theories," International Journal of Engineering Science, vol. 49, no. 11, pp. 1244-1255, 2011.

[20] R. Ansari and S. Sahmani, "Surface stress effects on the free vibration behavior of nanoplates," International Journal of Engineering Science, vol. 49, no. 11, pp. 1204-1215, 2011.

[21] D. Karličić, M. Cajić, and M. Stamenković, "Nonlinear vibration of nonlocal Kelvin-Voigt viscoelastic nanobeam embedded in elastic medium," in Proceedings of the 8th European Nonlinear Dynamics Conference (ENOC '14), Vienna, Austria, July 2014.

[22] E. Winkler, Theory of Elasticity and Strength, Dominicus, Prague, Czech Republic, 1867. 

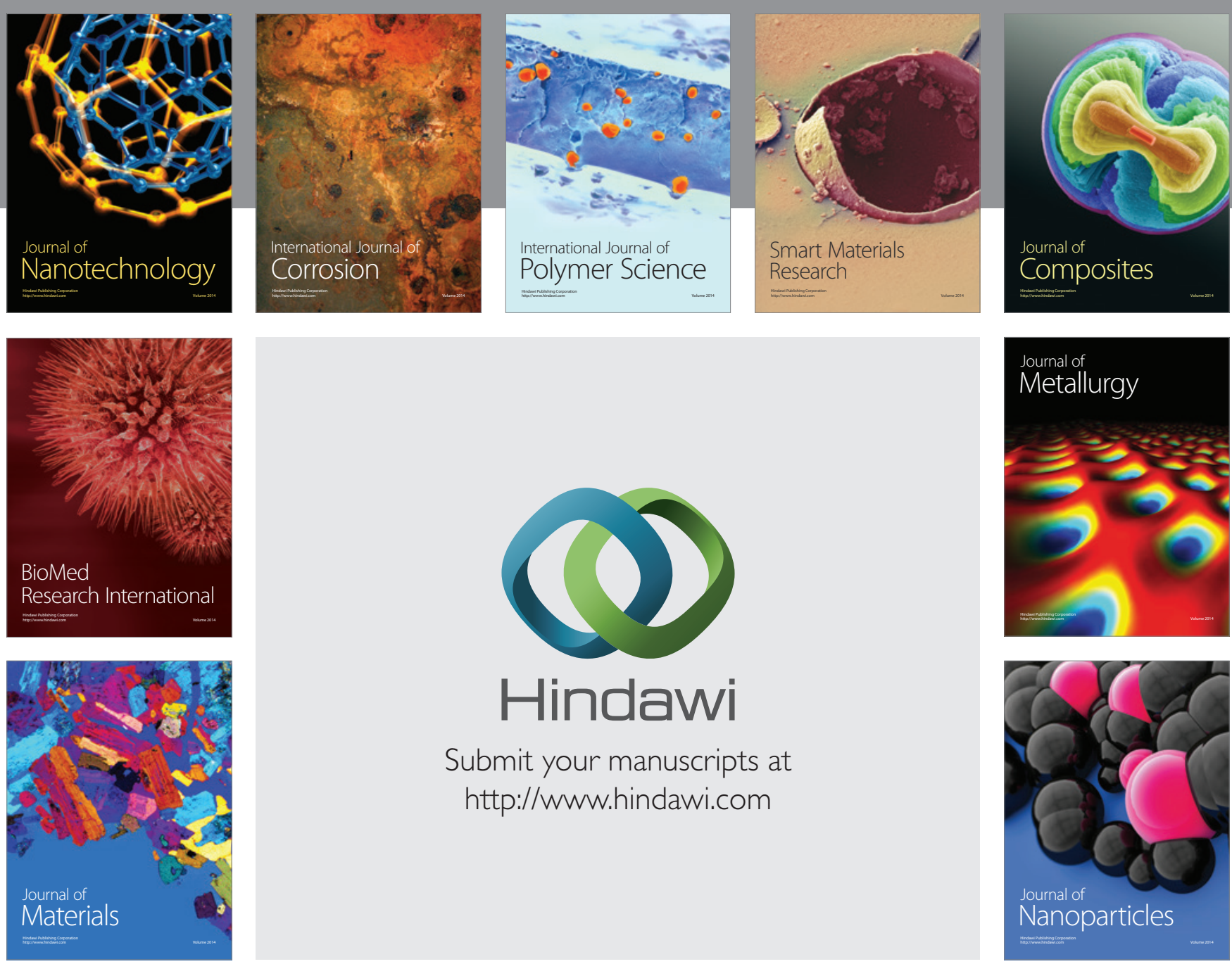

Submit your manuscripts at http://www.hindawi.com
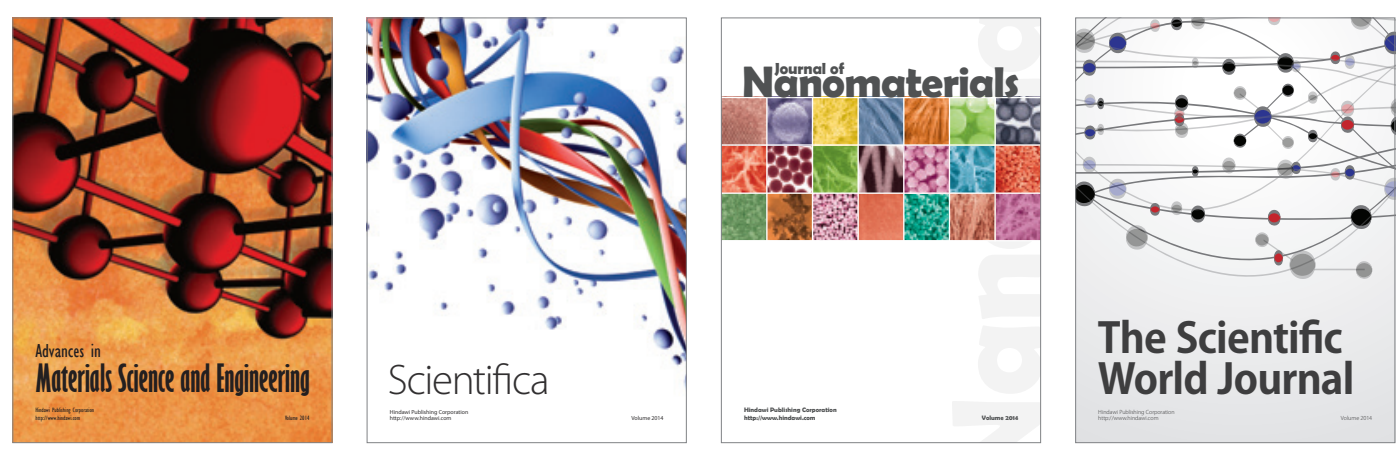

\section{The Scientific World Journal}
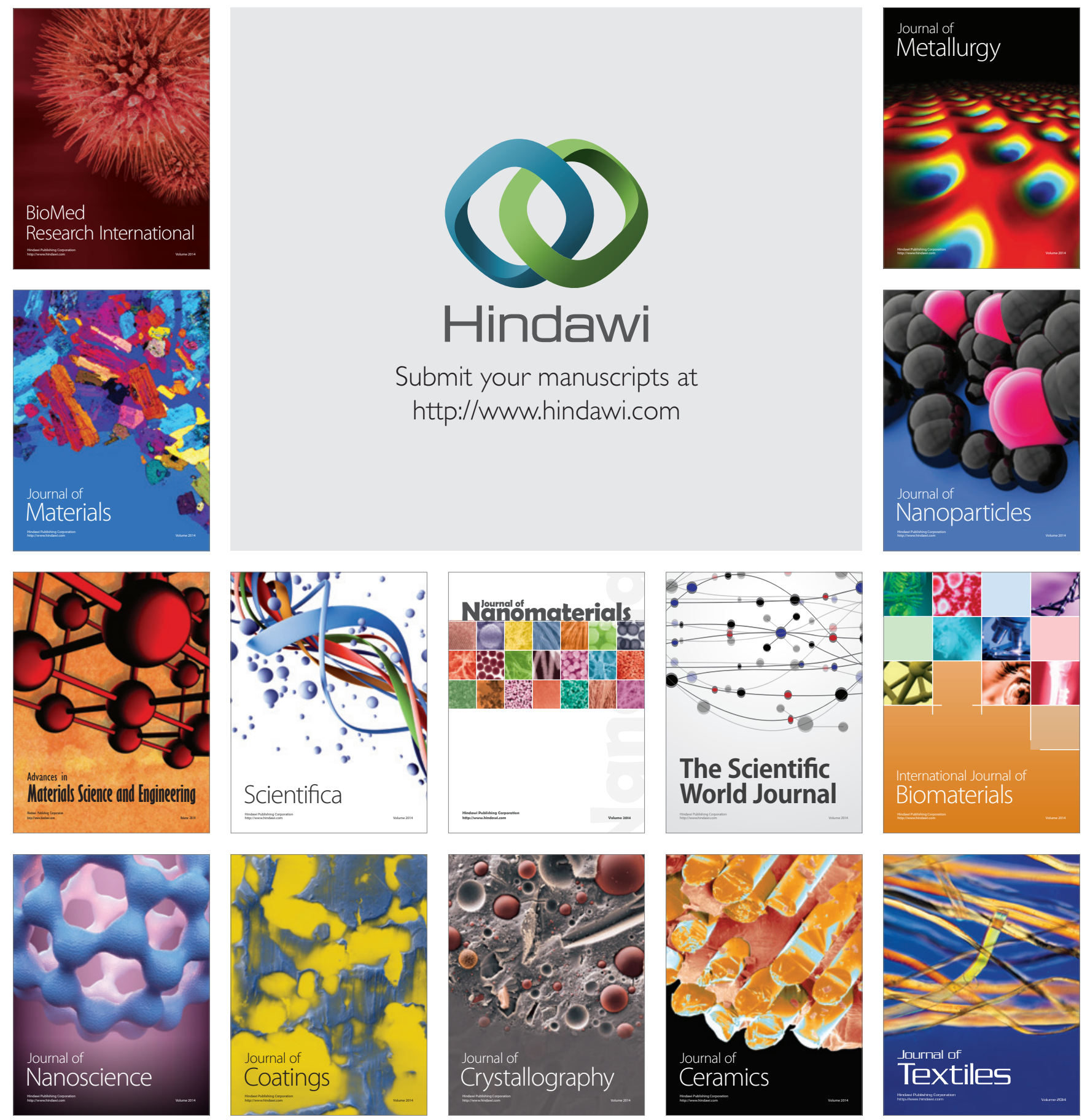